\title{
Equivariant periodicity for compact group actions
}

\author{
Shmuel Weinberger* and Min Yan ${ }^{\dagger}$
}

(Communicated by J. G. Ratcliffe)

\begin{abstract}
For a manifold $M$, the structure set $S(M$, rel $\partial)$ is the collection of manifolds homotopy equivalent to $M$ relative to the boundary. Siebenmann [3] showed that in the topological category, the structure set is 4-periodic: $S(M, \operatorname{rel} \partial) \cong S\left(M \times D^{4}\right.$, rel $\left.\partial\right)$ up to a copy of $\mathbb{Z}$. The periodicity has been extended to topological manifolds with homotopically stratified group actions for various representations in place of $D^{4}$, including twice any complex representation of a compact abelian group. In this paper, we extend the result to twice any complex representation of a compact Lie group. We also prove the bundle version of the periodicity.
\end{abstract}

\section{Introduction}

Probably the most basic structural phenomenon of high dimensional topology is Siebenmann's periodicity theorem [3] (as amended by Nicas [5]), which asserts that the manifolds homotopy equivalent to $M$ are in a one-to-one correspondence with (a subset of, because of nonresolvable homology manifolds [1]) those homotopy equivalent to $M \times D^{4}$. The main goal of this paper is to show the following extension of this to the equivariant setting.

Theorem. Let $G$ be a compact Lie group. Let $M$ be a homotopically stratified $G$ manifold that satisfies the codimension $>2$ gap condition. Let $\xi$ be a complex G-vector bundle over $M$ that has the same isotropy as $M$ everywhere. Then

$$
S_{G}(M, \operatorname{rel} \partial) \cong S_{G}(D(\xi \oplus \xi), \operatorname{rel} \partial)
$$

The codimension $>2$ gap condition means the following. Suppose $H \subset K$ are subgroups of $G$. Suppose $M_{\beta}^{K} \subset M_{\alpha}^{H}$ are equivariant components (connected components of the subsets of points fixed by the subgroups). Then either $M_{\beta}^{K}=M_{\alpha}^{H}$ or $\operatorname{dim} M_{\beta}^{K}+2<\operatorname{dim} M_{\alpha}^{H}$. The same isotropy everywhere condition means that for any

\footnotetext{
* Research was partially supported by an NSF grant.

${ }^{\dagger}$ Research was supported by Hong Kong Research Grant Council Earmark Grants HKUST6071/98P, HKUST6107/02P, and Direct Allocation Grant DAG01/02.SC20.
} 
open subset $U$ of $M$, the collection of isotropy groups for $U$ is the same as the collection of isotropy groups for $\left.\xi\right|_{U}$. The condition is equivalent to the condition that the projection induces a one-to-one correspondence between the isovariant components of $\xi$ and the isovariant components of $M$.

In fact, one can use topological bundles that have complex structure just over a 1skeleton on $M$. We plan to apply this, rather along the lines of Atiyah-Hirzebruch's differentiable Riemann-Roch theorem, in further joint work with Cappell to defining induced maps between structure sets for a wide class of equivariant maps. This should allow application of the tools of algebraic topology, such as homology theory and assembly maps, which do not generalize gracefully to the current situation where functoriality is only transparent for open inclusions.

This paper is a continuation of our earlier one on abelian group actions [7] and the reader can refer to the introduction to that paper for some more discussion of the context, history, and applications of such results, and also for any unexplained notation. In particular the main result of this paper proves a conjecture of that paper. For product bundles associated to representations (and some others), this result extends [8], which dealt with permutation representations of odd order groups, and [7] which dealt with abelian groups, and [9] which produced periodicity representations for some small positive dimensional nonabelian groups.

The proof of the main theorem uses the main technical device of [7]: the products with nonmanifold periodicity spaces. The main difference between this paper and that is the source of the periodicity space. In [7] we used products of modified projective spaces of representations of $G$. As [9] shows, this can occasionally be done in the nonabelian case, but it rapidly becomes unwieldy. Here we instead, following [2], modify configuration spaces of representation spheres to obtain the periodicity spaces. The basic idea is to consider the space of unordered pairs of elements of the unit sphere $S(V)$ of twice a complex representation. This space, although a nonmanifold, is a rational homology manifold stratified space, and the fixed set of every subgroup has signature 1 . Unfortunately, it has too many strata and the pure strata have excessive fundamental groups. The extra strata will be killed by constructing and attaching their "coboundaries". The excessive fundamental groups will be killed by a suitable equivariant surgery. The superior flexibility of the configuration space construction over projectivization enables one to handle nontrivial bundles, i.e. prove analogs of Thom isomorphism rather than just Bott periodicity. This very quickly leads to our main theorem.

This result was proven during a visit of the second author to the University of Chicago; he would like to thank them for their hospitality. The first author would like to thank Kevin Whyte for a valuable conversation. Both authors would also like to thank Sylvain Cappell for useful conversations regarding this work and its continuations.

\section{Periodicity space}

Let $V=W \oplus \mathbb{R}^{3}$ be the direct sum of a unitary complex $G$-representation $W$ and the trivial $G$-representation $\mathbb{R}^{3}$. We also fix two distinct vectors $p, q \in \mathbb{R}^{3}$ of unit 
length. Denote by $S(V)$ and $D(V)$ the unit sphere and the unit disk of the representation. Let

$$
C(V)=\frac{S(V) \times S(V)}{(u, v) \sim(v, u)}
$$

be the symmetric double of $S(V)$. Contained in $C(V)$ is the diagonal

$$
S_{\Delta}(V)=\{[v, v] \in C(V): v \in S(V)\}
$$

which is $G$-homeomorphic to $S(V)$. The $G$-space $C(V)$ is stratified with $S_{\Delta}(V)$ and $C(V)-S_{\Delta}(V)$ as the lower and upper strata. We use $p$ and $q$ to introduce a special point in the upper stratum

$$
b=[(0, p),(0, q)] \in C\left(\mathbb{R}^{3}\right)-S_{\Delta}\left(\mathbb{R}^{3}\right) \subset C\left(V^{G}\right)-S_{\Delta}\left(V^{G}\right) \subset C(V)-S_{\Delta}(V) .
$$

The tangent space at the point is

$$
T_{b} C(V)=T_{(0, p)} S\left(W \oplus \mathbb{R}^{3}\right) \times T_{(0, q)} S\left(W \oplus \mathbb{R}^{3}\right)=W \oplus \mathbb{R}^{2} \oplus W \oplus \mathbb{R}^{2}
$$

Our intention is to make $C(V)$ into a periodicity space, with the periodicity representation given by the tangent space above.

For a subgroup $H$ of $G$, we fix a finite generating set. Then $[v, w] \in C(V)^{H}$ means that for each generator $h$,

$$
\text { either } h v=v, h w=w, \quad \text { or } \quad h v=w, h w=v .
$$

Let $h_{1}, \ldots, h_{r}$ be the generators that fix $v$, and let $h_{1}^{\prime}, \ldots, h_{s}^{\prime}$ be the remaining generators that do not fix $v$ but exchange $v$ and $w$ instead. If $s=0$, then $H$ fixes $v$ and $w$, so that $[v, w] \in C\left(V^{H}\right)$. If $s>0$, then $v$ is fixed by the subgroup $K$ generated by $h_{i}$, $h_{j}^{\prime} h_{k}^{\prime}, h_{j}^{\prime} h_{i} h_{j}^{\prime}$. Moreover, $K$ is a normal subgroup of $H$ of index 2 . Thus if we let $h \in H$ be any element generating $H / K$ and denote

$$
S_{H, 2}\left(V^{K}\right)=\left\{[v, h v]: v \in S\left(V^{K}\right)\right\}
$$

then we have

$$
C(V)^{H}=C\left(V^{H}\right) \cup\left(\bigcup_{K \subset H,|H / K|=2} S_{H, 2}\left(V^{K}\right)\right) .
$$

By the way $K$ is constructed, the union is in fact a finite one. Moreover, the $W H$ action exchanges $S_{H, 2}\left(V^{K}\right)$ for various index 2 subgroups $K$ of $H$, and for $K \neq K^{\prime}$ we have

$$
S_{H, 2}\left(V^{K}\right) \cap S_{H, 2}\left(V^{K^{\prime}}\right)=S_{\Delta}\left(V^{H}\right)
$$


The space $C(V)$ cannot yet be a periodicity space for the following reasons:

(1) The singular part $S_{\Delta}(V)$ of $C(V)$ needs to be "killed";

(2) The extra fixed points $S_{H, 2}\left(V^{K}\right)$ needs to be "killed";

(3) The top stratum is not equivariantly simply connected: $\pi_{1}\left(C\left(V^{H}\right)-S\left(V^{H}\right)\right)=$ $\mathbb{Z}_{2}$.

To solve the first and the second problems, we need to find an equivariant "coboundary" for

$$
\hat{S}(V)=S_{\Delta}(V) \cup\left(\bigcup_{K \subset H \subset G,|H / K|=2} S_{H, 2}\left(V^{K}\right)\right) .
$$

It is easy to see that $\hat{S}(V)=\left\{[v, g v]: v \in S(V), g \in G, g^{2} v=v\right\}$. Moreover, we have the intersections among the pieces that make up $\hat{S}(V)$ :

$$
\begin{aligned}
S_{\Delta}(V) \cap S_{H, 2}\left(V^{K}\right) & =S_{\Delta}\left(V^{H}\right), \\
S_{\Delta}\left(V^{H}\right) \cap S_{\Delta}\left(V^{H^{\prime}}\right) & =S_{\Delta}\left(V^{H^{\prime \prime}}\right), \\
S_{H, 2}\left(V^{K}\right) \cap S_{H^{\prime}, 2}\left(V^{K^{\prime}}\right) & = \begin{cases}S_{\Delta}\left(V^{H^{\prime \prime}}\right) & \text { if } H^{\prime \prime}=K^{\prime \prime} \\
S_{H^{\prime \prime}, 2}\left(V^{K^{\prime \prime}}\right) & \text { if } H^{\prime \prime} \neq K^{\prime \prime},\end{cases}
\end{aligned}
$$

where $H^{\prime \prime}$ is the subgroup generated by $H, H^{\prime}$, and $K^{\prime \prime}$ is the subgroup generated by $K, K^{\prime}, h h^{\prime}$ ( $h$ and $h^{\prime}$ generate $H / K$ and $H^{\prime} / K^{\prime}$, respectively). The intersections imply that $\hat{S}(V)$ has a natural $G$-stratification with two types of closed strata, both labeled by conjugacy classes of isotropy subgroups. The first type is $(0$ stands for the lower strata, $[H]$ stands for the conjugacy class of $H$ )

$$
\hat{S}(V)_{0,[H]}=G S_{\Delta}\left(V^{H}\right),
$$

which gives the usual stratification of the $G$-sphere $S_{\Delta}(V)$ (any $G$-manifold has such natural $G$-stratification). The second type is (1 stands for the upper strata)

$$
\hat{S}(V)_{1,[H]}=G\left(S_{\Delta}\left(V^{H}\right) \cup\left(\bigcup_{K \subset H,|H / K|=2} S_{H, 2}\left(V^{K}\right)\right)\right) .
$$

The partial order among the strata is given by the inclusion of subgroups up to conjugation and the inclusion $\hat{S}(V)_{0,[H]} \subset \hat{S}(V)_{1,[H]}$.

Note that $v \leftrightarrow[v, v]$ is a homeomorphism between $S_{\Delta}(V)$ and $S(V)$, and $v \leftrightarrow[v, h v]$ is a homeomorphism between $S_{H, 2}\left(V^{K}\right)$ and $S\left(V^{K}\right) / H$ (which is really a quotient by $\left.H / K \cong \mathbb{Z}_{2}\right)$. Since $S_{\Delta}(V)$ and $S_{H, 2}\left(V^{K}\right)$ are spheres or quotients of spheres, we may construct the "coboundary" by extending the spheres to the disks. Specifically, inside the $G$-space 


$$
\frac{D(V) \times D(V)}{(u, v) \sim(v, u)}
$$

we introduce the subsets

$$
\begin{aligned}
D_{\Delta}(V) & =\{[v, v]: v \in D(V)\}, \\
D_{H, 2}\left(V^{K}\right) & =\left\{[v, h v]: v \in D\left(V^{K}\right)\right\},
\end{aligned}
$$

where $h \in H$ is any element generating $H / K$. The "coboundary" is then the subset

$$
\hat{D}(V)=D_{\Delta}(V) \cup\left(\bigcup_{K \subset H \subset G,|H / K|=2} D_{H, 2}\left(V^{K}\right)\right) .
$$

Moreover, similar homeomorphisms give us

$$
\begin{aligned}
D_{\Delta}(V) & \cong D(V)=\text { cone } S(V) \cong \text { cone } S_{\Delta}(V) \\
D_{H, 2}\left(V^{K}\right) & \cong D\left(V^{K}\right) / H=\operatorname{cone} S\left(V^{K}\right) / H \cong \text { cone } S_{H, 2}\left(V^{K}\right)
\end{aligned}
$$

which combine to form a homeomorphism of $G$-stratified spaces

$$
\hat{D}(V) \cong \text { cone } \hat{S}(V) \text {. }
$$

Note that all the strata of $\hat{S}(V)$ contain the minimum stratum $S\left(V^{G}\right)$, which is a sphere of dimension $\geqslant 2$ acted on trivially by the group. Therefore we may take the cone point in (2.5) not as a seperate stratum, but instead as an interior point of the closed stratum $D\left(V^{G}\right)$.

The cone relation (2.5) implies that the strata of $\hat{D}(V)$ and $\hat{S}(V)$ satisfy

$$
\hat{D}(V)^{\alpha}=\hat{S}(V)^{\alpha} \times(0,1], \quad \alpha=(0,[H]) \text { or }(1,[H]),
$$

with the pair $\left(D\left(V^{G}\right), S\left(V^{G}\right)\right)$ of smallest strata as the only exception. Consequently (together with $\operatorname{dim} V^{G} \geqslant 3$ ), the pair $(\hat{D}(V), \hat{S}(V))$ has the isovariant $\pi-\pi$ property.

Now we solve the problem that $\pi_{1}\left(C\left(V^{H}\right)-S\left(V^{H}\right)\right)$ is nontrivial. Take an embedding

$$
\rho: \mathbb{S}^{1} \rightarrow C\left(\mathbb{R}^{3}\right)-S_{\Delta}\left(\mathbb{R}^{3}\right)-\{b\}
$$

representing the generator of the fundamental group $\pi_{1}\left(C\left(\mathbb{R}^{3}\right)-S_{\Delta}\left(\mathbb{R}^{3}\right)\right)=\mathbb{Z}_{2}$. Since the space $C\left(\mathbb{R}^{3}\right)$ (which is homeomorphic to $\mathbb{C} P^{2}$ ) is orientable, the normal bundle of $\rho$ in $C\left(\mathbb{R}^{3}\right)$ is trivial. We fix one trivialization of the normal bundle.

The assumption $V=W \oplus \mathbb{R}^{3}$ allows us to naturally consider $S_{\Delta}\left(\mathbb{R}^{3}\right)$ and $C\left(\mathbb{R}^{3}\right)$ as subsets of $S_{\Delta}(V)$ and $C(V)$. The assumption also gives a natural trivialization of the normal bundle of $S\left(\mathbb{R}^{3}\right)$ in $S(V)$, with $W$ as the fibre. Since $\rho$ is contained in the top stratum $C(V)-\hat{S}(V)$, the normal bundle of $\rho$ in $C(V)$ can be naturally identified 


$$
v=\frac{[0,1] \times \mathbb{R}^{5} \times(W \oplus W)}{\left(0, u, w_{1}, w_{2}\right) \sim\left(1, u, w_{2}, w_{1}\right)} .
$$

Using the assumption that $W$ is a complex representation, a path

$$
\sigma_{t}=\left(\begin{array}{cc}
\cos \frac{\pi t}{2} & -e^{i \pi t} \sin \frac{\pi t}{2} \\
\sin \frac{\pi t}{2} & e^{i \pi t} \cos \frac{\pi t}{2}
\end{array}\right): W \oplus W \rightarrow W \oplus W
$$

may be constructed to connect the transformation $\left(w_{1}, w_{2}\right) \rightarrow\left(w_{2}, w_{1}\right)$ to the identity transformation in the space of equivariant automorphisms of $W \oplus W$. The path $\sigma_{t}$ can be interpreted as an explicit trivialization of the normal bundle (2.7), which can be used to identify a tubular neighborhood of $\rho$ in $C(V)$ with $\mathbb{S}^{1} \times D^{5} \times$ $D(W \oplus W) \cong \mathbb{S}^{1} \times D\left(\mathbb{R}^{5} \oplus W \oplus W\right)$. An equivariant surgery on $C(V)$ that replaces $\mathbb{S}^{1} \times D\left(\mathbb{R}^{5} \oplus W \oplus W\right)$ by $D^{2} \times S\left(\mathbb{R}^{5} \oplus W \oplus W\right)$ kills the fundamental groups $\pi_{1}\left(C\left(V^{H}\right)-S_{\Delta}\left(V^{H}\right)\right)$ for all $H$. We denote the result of the surgery by $C_{1}(V)$.

Since the surgery is performed inside $C(V)-\hat{S}(V)$, it can also be applied to (the top stratum of ) $C(V) \cup_{\hat{S}(V)} \hat{D}(V)$ and gives rise to

$$
P(V)=C_{1}(V) \cup_{\hat{S}(V)} \hat{D}(V) .
$$

To use $P(V)$ as a periodicity space, we need to verify that $C_{1}(V)^{H}-\hat{S}(V)^{H}=$ $C_{1}\left(V^{H}\right)-\hat{S}(V)^{H}$ (see $\left.(2.3)\right)$ is connected and simply connected for any subgroup $H$. Since the surgery already makes $C_{1}\left(V^{H}\right)-S_{\Delta}\left(V^{H}\right)$ connected and simply connected, it suffices to show that

$$
\operatorname{dim}\left(\hat{S}(V)^{H}-S_{\Delta}\left(V^{H}\right)\right)+3 \leqslant \operatorname{dim}\left(C(V)^{H}-S_{\Delta}\left(V^{H}\right)\right) .
$$

For the special case that $H$ is trivial, this means that

$$
\operatorname{dim}\left\{(v, g v): v \in V, g \in G, g v \neq v, g^{2} v=v\right\}+4 \leqslant 2 \operatorname{dim} V
$$

For a general subgroup $H$, we need to substitute $V$ and $G$ by $V^{H}$ and $W H$ in the inequality. Since $V$ already contains $\mathbb{R}^{3}$, the inequality is almost true (with +4 replaced by +3 ). The classical 4 -fold periodicity allows us to add $\mathbb{R}^{4}$ to $V$, so that $V \oplus \mathbb{R}^{4}$ satisfies the inequality. Therefore as far as proving the periodicity theorem is concerned, $P(V)$ can be taken as a periodicity space.

\section{Periodicity bundle}

In this section, a fibrewise construction of the periodicity space will be carried out. Attention is paid to the fact that the isotropy groups acting on fibres depend on the points of the base manifold. 
Let $M$ be a compact $G$-manifold. Let $\eta$ be a complex $G$-vector bundle over $M$. Let $\varepsilon^{3}=M \times \mathbb{R}^{3}$ be the three dimensional trivial bundle. Denote $\xi=\eta \oplus \varepsilon^{3}$ and the projection $\pi: \xi \rightarrow M$. For any subgroup $H, \xi^{H}$ is a $W H$-bundle over $M^{H}$.

We have the sphere bundle $S(\xi)$, the disk bundle $D(\xi)$, and the fibrewise symmetric product bundle $C(\xi)$ (which contains the diagonal bundle $S_{\Delta}(\xi)$ as lower stratum). All these are $G$-bundles.

Similar to the case $\xi=M \times V$, for any $K \subset H$ satisfying $|H / K|=2$, denote

$$
S_{H, 2}\left(\left.\xi^{K}\right|_{M^{H}}\right)=\left\{[v, h v]: v \in S\left(\left.\xi^{K}\right|_{M^{H}}\right)\right\}, \quad h \text { generates } H / K \text {. }
$$

Note that the bundle $\xi^{K}$ is restricted to $M^{H}$ because $v$ and $h v$ should lie in the same fibre of $S(\xi)$. Similar to $(2.3)$, the fixed point

$$
C(\xi)^{H}=C\left(\xi^{H}\right) \cup\left(\bigcup_{K \subset H,|H / K|=2} S_{H, 2}\left(\left.\xi^{K}\right|_{M^{H}}\right)\right) .
$$

The $W H$-action exchanges $S_{H, 2}\left(\left.\xi^{K}\right|_{M^{H}}\right)$ for various $K$, and by (2.4),

$$
S_{H, 2}\left(\left.\xi^{K}\right|_{M^{H}}\right) \cap S_{H, 2}\left(\left.\xi^{K^{\prime}}\right|_{M^{H}}\right)=S_{\Delta}\left(\xi^{H}\right), \quad \text { for } K \neq K^{\prime}
$$

To make (3.2) into a periodicity bundle, a "coboundary" for

$$
\begin{aligned}
\hat{S}(\xi) & =S_{\Delta}(\xi) \cup\left(\bigcup_{K \subset H \subset G,|H / K|=2} S_{H, 2}\left(\left.\xi^{K}\right|_{M^{H}}\right)\right) \\
& =\left\{[v, g v]: v \in S(\xi), g \in G_{\pi(v)}, g^{2} v=v\right\}
\end{aligned}
$$

needs to be constructed. The space $\hat{S}(\xi)$ is $G$-stratified with strata

$$
\begin{aligned}
& \hat{S}(\xi)_{0,[H]}=G S_{\Delta}\left(\xi^{H}\right), \\
& \hat{S}(\xi)_{1,[H]}=G\left(S_{\Delta}\left(\xi^{H}\right) \cup\left(\bigcup_{K \subset H,|H / K|=2} S_{H, 2}\left(\left.\xi^{K}\right|_{M^{H}}\right)\right)\right),
\end{aligned}
$$

and the projection $\hat{\pi}: \hat{S}(\xi) \rightarrow M$ is a stratified system of $G$-bundles, with $\hat{\pi}^{-1}(x)=$ $\hat{S}\left(\xi_{x}\right)$, where $\xi_{x}=\eta_{x} \oplus \mathbb{R}^{3}$ is a $G_{x}$-representation. Similar to (2.5), a fibrewise cone construction

$$
\hat{D}(\xi)=\text { cone }_{M} \hat{S}(\xi)=\hat{S}(\xi) \times[0,1] \cup M
$$

may be carried out, with $\hat{S}(\xi) \times 0$ glued to $M$ via the natural projection $\hat{\pi}$, and the cone point not introducing any extra stratum. By the same reason as before, the pair $(\hat{D}(\xi), \hat{S}(\xi))$ (and the restriction on any $G$-invariant subset of $M$ ) has the isovariant $\pi-\pi$ property. 
Next, the fibrewise fundamental group $\pi_{1}\left(C\left(\xi_{x}^{H}\right)-S_{\Delta}\left(\xi_{x}^{H}\right)\right), H \subset G_{x}$, needs to be killed. To do this, the special loop (2.6) may be extended to an embedding

$$
\rho_{M}: M \times \mathbb{S}^{1} \rightarrow C\left(\varepsilon^{3}\right)-S_{\Delta}\left(\varepsilon^{3}\right)-M \times b \rightarrow C(\xi)-S_{\Delta}(\xi)-M \times b
$$

The normal bundle of the embedding $\rho_{M}$ is similar to $(2.7)$

$$
v_{M}=\frac{[0,1] \times \mathbb{R}^{5} \times(\eta \oplus \eta)}{\left(0, u, w_{1}, w_{2}\right) \sim\left(1, u, w_{2}, w_{1}\right)},
$$

Then a fibrewise surgery as before produces a periodicity bundle (more precisely, a stratified system of bundles)

$$
\pi_{P}: P(\xi)=C_{1}(\xi) \cup_{\hat{S}(\xi)} \hat{D}(\xi) \rightarrow M
$$

The periodicity representation is the fibrewise tangent bundle of the section $M \times b$ in the top stratum $C_{1}(\xi)-S_{\Delta}(\xi)$. The fibrewise tangent bundle is easily identified with $\eta \oplus \eta \oplus \varepsilon^{4}$, so that the corresponding disk bundle

$$
E(\xi)=D\left(\eta \oplus \eta \oplus \varepsilon^{4}\right)
$$

is embedded in the top stratum $C_{1}(\xi)-S_{\Delta}(\xi)$. The triviality of the fundamental groups $\pi_{1}\left(C\left(\xi_{x}^{H}\right)-S_{\Delta}\left(\xi_{x}^{H}\right)\right)$ then implies that the embedding $E(\xi) \rightarrow C_{1}(\xi)-S_{\Delta}(\xi)$ $=P(\xi)-\hat{D}(\xi)$ induces isomorphisms on the fundamental groups of the isovariant components.

\section{Periodicity for surgery obstruction}

Denote by $L_{G}$ the surgery obstruction space, with the homotopy groups being the surgery obstruction groups of Browder and Quinn. The periodicity is based on the fact that the following maps

$$
L_{G}(M) \stackrel{\operatorname{trf}}{\longrightarrow} L_{G}(P(\xi)) \stackrel{\text { incl }}{\longleftarrow} L_{G}(E(\xi))
$$

are homotopy equivalences.

The inclusion in (4.1) is the composition of the following inclusions

$$
L_{G}(E(\xi)) \stackrel{\text { incl }_{\alpha}}{\longrightarrow} L_{G}(P(\xi)-\hat{D}(\xi)) \stackrel{\text { incl }_{\beta}}{\longrightarrow} L_{G}(P(\xi)) .
$$

Since the embedding $E(\xi) \rightarrow P(\xi)-\hat{D}(\xi)$ induces isomorphisms on the fundamental groups of the isovariant components, $i n c l_{\alpha}$ is an equivalence. The second inclusion fits into a fibration

$$
L_{G}(P(\xi)-\hat{D}(\xi)) \stackrel{\text { incl }_{\beta}}{\longrightarrow} L_{G}(P(\xi)) \longrightarrow L_{G}(\hat{D}(\xi)) .
$$


The base is homotopically trivial because the pair $(\hat{D}(\xi), \hat{S}(\xi))$ has the isovariant $\pi-\pi$ property. Thus $\operatorname{incl}_{\beta}$ is also an equivalence. This completes the proof that the inclusion in (4.1) is an equivalence.

To prove that the transfer in (4.1) is an equivalence, consider a maximal isotropy subgroup $H$ of $M$. There is a commutative diagram

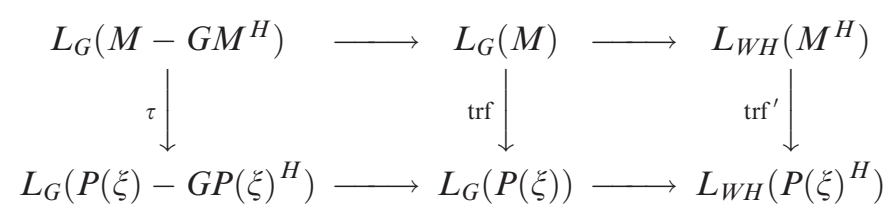

in which the rows are fibrations and the transfer map trf ${ }^{\prime}$ is taken with respect to the WH-bundle $P(\xi)^{H}=C_{1}(\xi)^{H} \cup \hat{D}(\xi)^{H} \rightarrow M^{H}$. We will prove the transfer map in the middle to be an equivalence by showing that the maps $\tau$ and $\operatorname{trf}^{\prime}$ are equivalences.

The transfer $\operatorname{trf}^{\prime}$ may be compared with a related non-equivariant transfer. Since $H$ is a maximal isotropy subgroup, $W H$ acts freely on the equivariant $W H$ bundle $\xi^{H} \rightarrow M^{H}$. Then we have the disk bundle $D\left(\xi^{H} / W H\right)$, the sphere bundle $S\left(\xi^{H} / W H\right)$, and the symmetric product $C\left(\xi^{H} / W H\right)$ of the sphere bundle. Moreover, a surgery can be applied to $C\left(\xi^{H} / W H\right)$ to get $C_{1}\left(\xi^{H} / W H\right)$ and a stratified system of bundles

$$
P\left(\xi^{H} / W H\right)=C_{1}\left(\xi^{H} / W H\right) \cup_{S_{\Delta}\left(\xi^{H} / W H\right)} D\left(\xi^{H} / W H\right) \rightarrow M^{H} / W H .
$$

Then there is a transfer map

$$
\operatorname{trf}^{\prime \prime}: L\left(M^{H} / W H\right) \rightarrow L\left(P\left(\xi^{H} / W H\right)\right)
$$

associated to the bundle. Moreover, there is an inclusion

$$
\operatorname{incl}^{\prime \prime}: L\left(C_{1}\left(\xi^{H} / W H\right)-S_{\Delta}\left(\xi^{H} / W H\right)\right) \rightarrow L\left(P\left(\xi^{H} / W H\right)\right)
$$

which is again an equivalence because $\left(D\left(\xi^{H} / W H\right), S_{\Delta}\left(\xi^{H} / W H\right)\right)$ has the $\pi-\pi$ property. Since the dimension of the fibre of $C\left(\xi^{H} / W H\right)$ is $2\left(\operatorname{dim} \xi^{H}-1\right)$, there is also the projection

$$
\text { proj : } L\left(C_{1}\left(\xi^{H} / W H\right)-S_{\Delta}\left(\xi^{H} / W H\right)\right) \rightarrow L_{+2\left(\operatorname{dim} \xi^{H}-1\right)}\left(M^{H} / W H\right),
$$

which is also an equivalence because the projection induces an isomorphism on fundamental groups. Moreover, since $\xi^{H}=\eta^{H} \oplus \varepsilon^{3}$ and $\eta$ is a complex vector bundle, $\operatorname{dim} \xi^{H}-1$ is an even number, and we have the classical periodicity isomorphism

$$
\text { per : } L_{+2\left(\operatorname{dim} \xi^{H}-1\right)}\left(M^{H} / W H\right) \cong L\left(M^{H} / W H\right)
$$


Combining the four maps associated to the bundle $P\left(\xi^{H} / W H\right)$ together, we get

$$
\text { per } \circ \text { proj } \circ \text { incll-1 }^{\prime \prime} \circ \operatorname{trf}^{\prime \prime}: L\left(M^{H} / W H\right) \rightarrow L\left(M^{H} / W H\right) \text {. }
$$

Since the complex vector bundle $\xi^{H} / W H$ is always orientable and even dimensional, the proof of Theorem 4.3 in [2] basically says that the composition (4.6) is an equivalence. Since the periodicity, the projection, and the inclusion are all equivalences, the transfer (4.4) is also an equivalence.

Next the two transfers $\operatorname{trf}^{\prime}$ and trf ${ }^{\prime \prime}$ are compared. By (3.2) and (3.3),

$$
\begin{aligned}
C_{1}(\xi)^{H} & =C_{1}\left(\xi^{H}\right) \cup S_{H}, \\
\hat{S}(\xi)^{H} & =S_{\Delta}\left(\xi^{H}\right) \cup S_{H}, \\
P(\xi)^{H} & =\left(C_{1}\left(\xi^{H}\right) \cup S_{H}\right) \cup_{\left(S_{\Delta}\left(\xi^{H}\right) \cup S_{H}\right)}\left(D_{\Delta}\left(\xi^{H}\right) \cup D_{H}\right),
\end{aligned}
$$

where

$$
S_{H}=\bigcup_{K \subset H,|H / K|=2} S_{H, 2}\left(\left.\xi^{K}\right|_{M^{H}}\right), \quad D_{H}=\bigcup_{K \subset H,|H / K|=2} D_{H, 2}\left(\left.\xi^{K}\right|_{M^{H}}\right) .
$$

Moreover, by the way $P\left(\xi^{H} / W H\right)$ is constructed,

$$
\begin{aligned}
C_{1}\left(\xi^{H} / W H\right) & =C_{1}\left(\xi^{H}\right) / W H, \\
S_{\Delta}\left(\xi^{H} / W H\right) & =S_{\Delta}\left(\xi^{H}\right) / W H, \\
P\left(\xi^{H} / W H\right) & =\left(C_{1}\left(\xi^{H}\right) \cup_{S_{\Delta}\left(\xi^{H}\right)} D_{\Delta}\left(\xi^{H}\right)\right) / W H .
\end{aligned}
$$

Thus the only difference between $P\left(\xi^{H} / W H\right)$ and the quotient $P(\xi)^{H} / W H$ are the extra strata $D_{H} / W H$ and $S_{H} / W H$. Forgetting the extra strata gives a commutative diagram

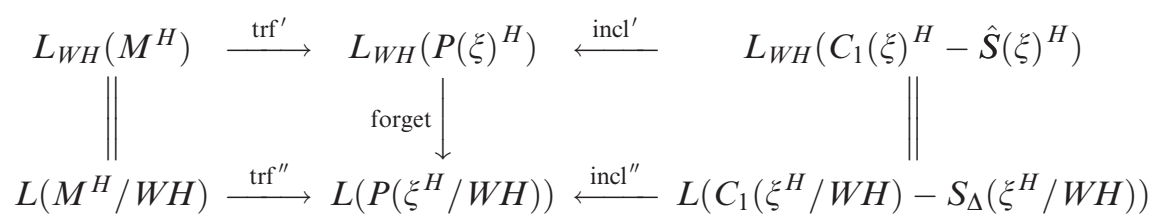

It has been argued before that incl" in (4.5) is an equivalence. By the similar $\pi-\pi$ reason, the inclusion incl' is also an equivalence. Consequently, the transfer $\operatorname{trf}^{\prime}$ is an equivalence.

Now consider the induced map $\tau$ in (4.3). The map fits into a commutative diagram 


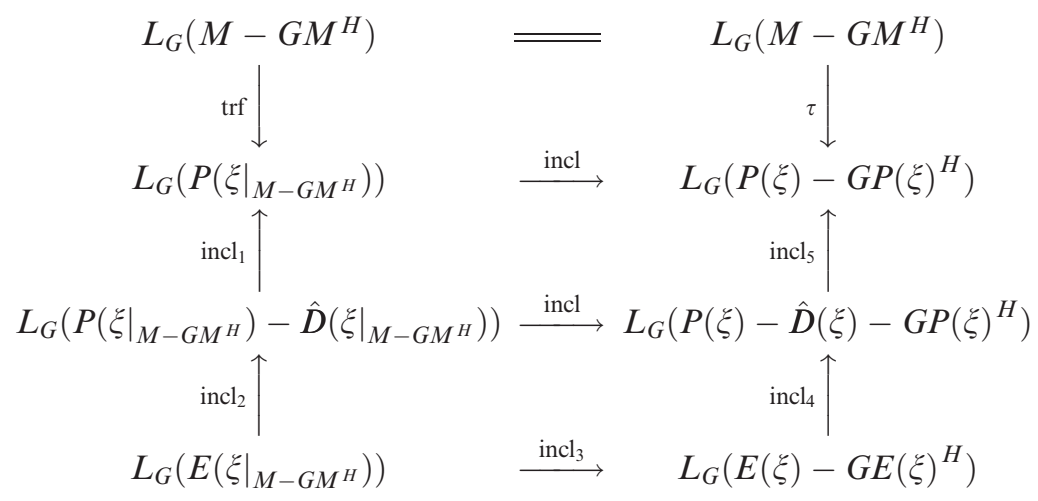

Since $M-G M^{H}$ has fewer isotropy subgroups than $M$, it can be assumed, by induction, that the transfer on the top left is already an equivalence. Then $\tau$ can be proved to be an equivalence if the indexed inclusions incl $_{1}, \ldots$, incl $_{5}$ can be shown to be equivalences.

The composition of incl $_{1}$ and incl $_{2}$ is the same as the composition in (4.2), except $M$ is replaced by $M-G M^{H}$. Moreover, because $G E(\xi)^{H}=E(\xi) \cap G P(\xi)^{H}$, the composition of incl $_{4}$ and incl $_{5}$ is the same as (4.2), except the isovariant components of (the maximal isotropy subgroup) $H$ and its conjugates are deleted. The modifications do not affect the arguments for the inclusions in (4.2) to be equivalences. Therefore by the same arguments, the inclusion maps incl 4 , incl 5 are equivalences.

Finally, consider the spaces $E\left(\left.\xi\right|_{M-G M^{H}}\right)$ and $E(\xi)-G E(\xi)^{H}$ on the two sides of incl $_{3}$. Denote $\zeta=\eta \oplus \eta \oplus \varepsilon^{4}$. Then

$$
\begin{aligned}
E\left(\left.\xi\right|_{M-G M^{H}}\right) & =D\left(\left.\zeta\right|_{M-G M^{H}}\right), \\
E(\xi)-G E(\xi)^{H} & =D(\zeta)-G D(\zeta)^{H} \\
& =D\left(\left.\zeta\right|_{M-G M^{H}}\right) \cup G \times_{H}\left(D\left(\left.\zeta\right|_{M^{H}}\right)-D\left(\zeta^{H}\right)\right) .
\end{aligned}
$$

For any proper subgroup $K$ of $H$, the following is a one-to-one correspondence:

- the $K$-isovariant components of $M$ and of $M-G M^{H}$, via inclusion.

The same isotropy everywhere assumption implies the following are one-to-one correspondences:

- the $K$-isovariant components of $D(\zeta)$ and of $M$, via projection;

- the $K$-isovariant components of $D\left(\left.\zeta\right|_{M-G M^{H}}\right)$ and of $M-G M^{H}$, via projection.

Combining the three one-to-one correspondences, we conclude that inclusion gives a one-to-one correspondence between the $K$-isovariant components of $D(\zeta)$ and of $D\left(\left.\zeta\right|_{M-G M^{H}}\right)$. Then by the codimension $\geqslant 3$ gap condition, the corresponding $K$ isovariant components of $D(\zeta)$ and of $D\left(\left.\zeta\right|_{M-G M^{H}}\right)$ have the same fundamental groups (same as the fundamental groups of the corresponding isovariant components of $M$ and $\left.M-G M^{H}\right)$. Since $K$-isovariant components of $D(\zeta)$ are the same as the 
$K$-isovariant components of $D(\zeta)-G D(\zeta)^{H}$, we conclude that incl $_{3}$ induces isomorphisms on the fundamental groups of the corresponding isovariant components. Consequently, incl 3 induces an equivalence on the surgery obstruction.

\section{Periodicity for $K$-theory}

The discussion of the third section of [9] is still valid here. There are two periodicity problems concerning the $K$-theory. The first is the stabilization of the surgery obstruction. The second is the destabilization of the structure set. By mostly formal arguments, both problems can be settled by considering the special case where $G$ acts freely on $M$ and by considering the diagram

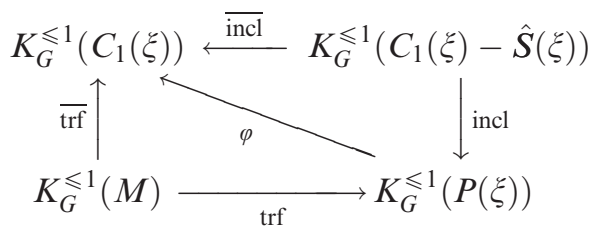

in which $C_{1}(\xi)$ is no longer considered as stratified, but only as a $G$-bundle over $M$ with polyhedron $C_{1}(V)$ as the fibre. The map $\varphi$ is the restriction to the closed stratum $C_{1}(\xi)$ followed by forgetting the stratification in $C_{1}(\xi)$. The geometrical meanings of the maps in (5.1) imply that the two triangles (and hence the whole diagram) are commutative. Since incl induces isomorphisms on the fundamental groups of isovariant components, it is an equivalence on the $K$-theory. As argued in [9], what needs to be done is to show that $\overline{\operatorname{trf}}$ is an equivalence after localizing at 2 . In fact, we are going to see that the transfer is the multiplication by 3 .

Since $G$ acts freely, $\overline{\operatorname{trf}}$ is the same as the transfer $K^{\leqslant 1}(M / G) \rightarrow K^{\leqslant 1}\left(C_{1}(\xi) / G\right)$ for the nonequivariant bundle $C_{1}(\xi) / G \rightarrow M / G$, which was studied in [4]. Fix a base point of $C_{1}(\xi) / G$ and use its projection $b=G x(x \in M)$ as the base point of $M / G$. Denote by $V=\xi_{x}$ the fibre of $\xi$ over $x$. Then $C_{1}(V)$ is the fibre of the bundle $C_{1}(\xi) / G \rightarrow M / G$, and the universal cover $\tilde{C}_{1}(\xi)$ of the total space $C_{1}(\xi) / G$ is given by the following pullbacks

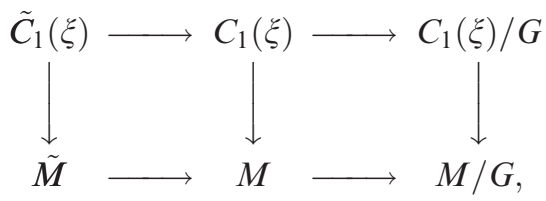

where $\tilde{M}$ is the universal cover of $M$. Since $C_{1}(V)$ is connected and simply connected, $\pi_{1}\left(C_{1}(\xi) / G\right)$ may be identified with $\pi_{1}(M / G)$ via the projection. Then by fixing a base point $\tilde{b} \in \tilde{M}$ above $b$, the fibre of $\tilde{M} \rightarrow M / G$ is exactly $\pi_{1}(M / G) \tilde{b}$. From the pullback (5.2), the fibre $\tilde{C}_{1}(V)$ of the bundle $\tilde{C}_{1}(\xi) \rightarrow M / G$ is then exactly $\pi_{1}(M / G) \tilde{b} \times C_{1}(V)$ as a $\pi_{1}(M / G)$-space. The monodromy of the $\pi_{1}(M / G)$ equivariant bundle $\tilde{M} \rightarrow M / G$ then gives rise to a homomorphism 


$$
\pi_{1}(M / G) \rightarrow\left[\tilde{C}_{1}(V), \tilde{C}_{1}(V)\right]_{\pi_{1}(M / G)},
$$

where $[\tilde{F}, \tilde{F}]_{\pi}$ denotes the equivalent homotopy classes of self-homotopy equivalences of a $\pi$-space $\tilde{F}$. Note that in case $\tilde{F}=\pi \times F$, with the $\pi$-action being left multiplication on the first and the trivial action on the second factor, we have a natural map

$$
[F, F] \rightarrow[\tilde{F}, \tilde{F}]_{\pi}, \quad \tilde{\varphi}(g, x)=(g, \varphi(x)) .
$$

Now we are in exactly the same situation. The pullback (5.2) implies that the homomorphism (5.3) is a composition

$$
\pi_{1}(M / G) \rightarrow\left[C_{1}(V), C_{1}(V)\right] \rightarrow\left[\tilde{C}_{1}(V), \tilde{C}_{1}(V)\right]_{\pi_{1}(M / G)},
$$

where the first map is the monodromy of the bundle $C_{1}(\xi) / G \rightarrow M / G$, and the second map is (5.4).

By Theorem 2.1 of the second part of [4], the transfer $K^{\leqslant 1}(M / G) \rightarrow$ $K^{\leqslant 1}\left(C_{1}(\xi) / G\right)$ is algebraically determined by the $\mathbb{Z} \pi_{1}(M / G)-\mathbb{Z} \pi_{1}(M / G)$-bimodule structure on the homologies $H_{i}\left(\tilde{C}_{1}(V)\right)$ induced by the homomorphism (5.3). The first $\mathbb{Z} \pi_{1}(M / G)$ refers to the group $\pi_{1}(M / G)$ on the right (appearing as a subscript) of (5.3). The second $\mathbb{Z} \pi_{1}(M / G)$ refers to the group $\pi_{1}(M / G)$ on the left of (5.3). Since both $\pi_{1}(M / G)$ in $(5.3)$ were considered as acting on the left of $\tilde{C}_{1}(V)$, the action of the group $\pi_{1}(M / G)$ on the left of (5.3) needs to be modified by the inverse in the bimodule structure.

In our case, the factorization (5.5) implies that

$$
H_{i}\left(\tilde{C}_{1}(V)\right)=\mathbb{Z} \pi_{1}(M / G) \otimes H_{i}\left(C_{1}(V)\right) .
$$

Moreover, the bimodule structure is the following: the first $\mathbb{Z} \pi_{1}(M / G)$ acts by left multiplication on the first factor only. The second $\mathbb{Z} \pi_{1}(M / G)$ acts on the second factor only, and is induced by the monodromy of the bundle $C_{1}(\xi) / G \rightarrow M / G$.

Since $H_{i}\left(C_{1}(V)\right)$ is a finitely generated abelian group, $H_{i}\left(\tilde{C}_{1}(V)\right)$ has finitely generated projective resolutions as (the first and the left) $\mathbb{Z} \pi_{1}(M / G)$-module. Thus according to Theorem 2.1 of the second part of [4], the transfer is determined by the element

$$
\sum(-1)^{i}\left[H_{i}\left(\tilde{C}_{1}(V)\right)\right]=\left[\mathbb{Z} \pi_{1}(M / G)\right] \otimes \sum(-1)^{i}\left[H_{i}\left(C_{1}(V)\right)\right]
$$

in a certain Grothendieck group $K_{0}\left(\mathbb{Z} \pi_{1}(M / G)-\mathbb{Z} \pi_{1}(M / G)\right)$.

Let $2 k-2$ be the real dimension of the complex bundle $\eta$. Then $\xi$ is a real orientable bundle of real dimension $2 k+1$. Based on the computation in [2] (see the discussion on page 299 leading to 4.8), it is easy to see that

$$
H_{i}\left(C_{1}(V)\right)=\left\{\begin{array}{ll}
H_{i}\left(\mathbb{S}^{2 k}\right) & i=0,2 k \\
0 & i=1 \\
\tilde{H}_{i-2 k-1}\left(\mathbb{R P}^{2 k-1}\right) & \text { other } i
\end{array}= \begin{cases}\mathbb{Z} & i=0,2 k, 4 k \\
\mathbb{Z}_{2} & i=3,5, \ldots, 2 k-1 \\
0 & \text { other } i\end{cases}\right.
$$


as abelian groups. Since $\xi$ is orientable, the monodromy preserves the orientation of the fibre. Then by tracing the computation in [2] (see the discussion at the bottom of page 299 leading to 4.10), we find the action of $\mathbb{Z} \pi_{1}(M / G)$ on $H_{i}\left(C_{1}(V)\right)$ to be trivial. Thus the element (5.6) is the same as the element $\left[\mathbb{Z} \pi_{1}(M / G)\right] \otimes\left[\mathbb{Z}^{3}\right]=$ $3\left[\mathbb{Z} \pi_{1}(M / G)\right]$ in the Grothendieck group $K_{0}\left(\mathbb{Z} \pi_{1}(M / G)-\mathbb{Z} \pi_{1}(M / G)\right)$. This implies that $\operatorname{trf}$ is multiplication by 3 .

\section{References}

[1] J. Bryant, S. Ferry, W. Mio, S. Weinberger, Topology of homology manifolds. Ann. of Math. (2) 143 (1996), 435-467. MR1394965 (97b:57017) Zbl 0867.57016

[2] F. T. Farrell, L. E. Jones, A topological analogue of Mostow's rigidity theorem. J. Amer. Math. Soc. 2 (1989), 257-370. MR973309 (90h:57023a) Zbl 0696.57018

[3] R. C. Kirby, L. C. Siebenmann, Foundational essays on topological manifolds, smoothings, and triangulations. Princeton Univ. Press 1977. MR0645390 (58 \#31082) Zbl 0361.57004

[4] W. Lück, The transfer maps induced in the algebraic $K_{0}$ - and $K_{1}$-groups by a fibration. I. Math. Scand. 59 (1986), 93-121. MR873491 (88d:18007) Zbl 0589.57020

[5] A. J. Nicas, Induction theorems for groups of homotopy manifold structures. Mem. Amer. Math. Soc. 39 (1982), vi+108. MR668807 (83i:57026) Zbl 0507.57018

[6] S. Weinberger, The topological classification of stratified spaces. University of Chicago Press, Chicago, IL 1994. MR1308714 (96b:57024) Zbl 0826.57001

[7] S. Weinberger, M. Yan, Equivariant periodicity for abelian group actions. Adv. Geom. 1 (2001), 49-70. MR1823952 (2002f:57050) Zbl 0980.57009

[8] M. Yan, The periodicity in stable equivariant surgery. Comm. Pure Appl. Math. 46 (1993), 1013-1040. MR1223661 (95d:57017) Zbl 0833.57020

[9] M. Yan, Equivariant periodicity in surgery for actions of some nonabelian groups. In: Geometric topology (Athens, GA, 1993), volume 2 of AMS/IP Stud. Adv. Math., 478-508, Amer. Math. Soc. 1997. MR1470745 (98f:57057) Zbl 0896.55010

Received 3 December, 2003

S. Weinberger, Department of Mathematics, University of Chicago, Chicago IL 60637, USA Email: shmuel@math.uchicago.edu

M. Yan, Department of Mathematics, Hong Kong University of Science and Technology Email: mamyan@ust.hk 
Copyright of Advances in Geometry is the property of Walter de Gruyter GmbH \& Co. KG.. The copyright in an individual article may be maintained by the author in certain cases. Content may not be copied or emailed to multiple sites or posted to a listserv without the copyright holder's express written permission. However, users may print, download, or email articles for individual use. 